\title{
Generalized isochrone models for spherical stellar systems *
}

\author{
G.G. KUZMIN and Ü.-I.K. VELTMANN
}

Tartu Astrophysical Observatory, Estonia

The space density $\rho(r)$ of a spherical system with potential $\Phi(r)$ is related to the phase density $\Psi(x, \xi)$ as a function of specific energy $x$ and angular momentum $2 \xi$ by a well-known integral equation. If the function $\rho(r)$ is given (and thus also $\Phi(r)$ ), this integral equation has no unique solution. In order to transform this problem into one with a unique solution we suggest the following form for $\Psi(x, \xi)$ :

$$
\Psi(x, \xi)=\Theta^{-\mu} \psi(u), \quad u=\frac{x-\lambda \xi}{\Theta}, \quad \Theta=1+p \xi
$$

where $\mu$ (dimensionless), $\lambda$ and $p$ are parameters satisfying $p \geq 0$ and $\lambda \geq r_{\text {max }}^{-2}$, with $r_{\text {max }}$ the limiting radius of the star system. The additive constant in the potential is chosen so that $\Phi\left(r_{\max }\right)=0$. To make the parameters $\lambda$ and $p$ dimensionless and give them some meaning we adopt the central potential $\Phi(0)$ as the unit of $\Phi, x$ and $u$, and the equivalent radius of the stellar system $r_{e}$ as the unit of length. Then $\Phi(0) r_{e}^{2}$ will be the unit of $\xi$. We obtain the integral equation

$$
g(\Phi)=4 \sqrt{2} \pi \int_{0}^{\Phi}(\Phi-u)^{\frac{1}{2}} F(1,5 / 2-\mu, 3 / 2 ; p s) \psi(u) d u
$$

for the unknown function $\psi(u)$ which we call the partial density. The function $F$ is the Gauss hypergeometric function,

$$
g(\Phi):=\left[1+(\lambda+\rho \Phi) r^{2}(\Phi)\right] \rho
$$

and

$$
s(\Phi, u)=\frac{(\Phi-u) r^{2}(\Phi)}{1+(\lambda+\rho \Phi) r^{2}(\Phi)} .
$$

If we put $\mu=5 / 2$ and $\lambda=p=0$, then the equation (2) is of the well-known Eddington (1916) form:

$$
g(\Phi)=4 \sqrt{2} \pi \int_{0}^{\Phi}(\Phi-u)^{\frac{1}{2}} \Psi(u) d u .
$$

In this case $g(\Phi)=\rho(\Phi)$. If $g(\Phi)$ is represented as a power series in $\Phi$, then it is easy to obtain the solution in the form of a power series in $u$, the term with $\Phi^{n}$ corresponding to the term $u^{n-\frac{3}{2}}$. Let us suppose we have an expansion ( finite of infinite)

$$
g(\Phi)=\sum_{m, n} g_{m, n} \frac{\Phi^{n}}{(1-q \Phi)^{n}}
$$

* Originally published in: T.B. Omarov (ed.), Dynamics of Galaxies and star clusters, "Nauka"Kaz.SSR, Alma-Ata, 1973, p.82-87, Translated by L.P. Ossipkov. 
with $q$ a parameter. Then we obtain the solution in the form

$$
\psi(u)=\sum_{m, n} \psi_{m, n} F\left(m, n+1, n-\frac{1}{2} ; q u\right) u^{n-\frac{3}{2}}
$$

where

$$
\psi_{m, n}=g_{m, n}[4 \sqrt{2} \pi B(n-1 / 2,3 / 2)]^{-1} .
$$

In the simplest case $g(\Phi)$ is proportional to $\Phi^{n}$. If moreover $\lambda=p=0$ then $\rho$ is proportional to $\Phi^{n}$ and we recover the isotropic polytropes. If, on the other hand, $\lambda \neq 0$ but $p=0$ then we find the quasi-polytropes studied by Camm (1952) and Idlis (1961). is

Now let us consider generalised isochrone models. The potential of these models

$$
\Phi(r)=a(b+\zeta)^{-1}, \quad \zeta(r)=\left(1+a^{2} r^{2}\right)^{\frac{1}{2}}, \quad a=b+1 .
$$

Adopting $M / \frac{4}{3} r_{e}^{3}$ as the unit of the density, where $M$ is the mass of the stellar system, we find the following density

$$
\rho(r)=\frac{1}{3} a^{3}\left(b+3 \zeta+2 b \zeta^{2}\right)(b+\zeta)^{-3} \zeta^{-3} .
$$

It is evident that the function $g(\Phi)$ can be represented in the form of the expansion (6), and $q=b / a$. In the simplest case of the Schuster model $b=0=q=0$ ) the expansion (6) is reduced to a polynomial. We obviously recover a polynomial for $\psi(u)$ :

$$
\psi(u)=\frac{2 \sqrt{2}}{\pi^{2}}\left[\lambda+\frac{8}{5} p u+\frac{16}{7}(1-\lambda) u^{2}-\frac{64}{21} p u^{3}\right] u^{\frac{3}{2}} .
$$

If $\lambda=1, p=0$ or $\lambda=0, p>0$ we obtain models considered in KK68b, resp KK68a.

In general the expansion (6) consists of six terms. Let us restrict ourselves for sake of simplicity to $\lambda=0$. Then there will be only five terms in the expansion (6) and the coefficients will have a more simple form. The expansion is not unique of course. If we fix $n=3$ then the coefficients of the expansion (2) will be the following:

$$
\begin{aligned}
g_{-1,3} & =\frac{1}{3}\left(1-b^{-2}\right) p q, \quad g_{0,3}=\frac{1}{3}\left(1-b^{-2}\right)\left(-2+5 b^{-2} p q\right) \\
g_{1,3} & =\frac{1}{3}\left[2-3 b^{-2}-b^{-2}\left(1-3 b^{-2}\right) p q\right], \quad g_{2,3}=\frac{1}{3} b^{-4} p q \\
g_{3,3} & =\frac{1}{3} b^{-2}\left(1-b^{-2} p q\right) .
\end{aligned}
$$

These coefficients are to be multiplied by the same factor $2 \sqrt{2} \pi^{-2}$ for the transition to the expansion (7). It is evident that the first two hypergeometrical functions will yield only linear expression in $u$, so only three non-trivial hypergeometrical functions remain.

For the limiting model $b=\infty,(q=1)$ only one non-trivial hypergeometrical function will appear. We have 


$$
\psi(u)=\frac{4 \sqrt{2}}{3 \pi^{2}}\left[F(1,4,5 / 2 ; u)-1-p\left(1-\frac{8}{5} u\right)\right] .
$$

For the isochroneous model $b=1,\left(q=\frac{1}{2}\right)$ the solution is reduced to the formula (13.11) of KK68a. Three hypergeometrical functions can be found by recursion from

$$
F(1,1,3 / 2, z)=\frac{\arcsin (\sqrt{z})}{\sqrt{z(1-z)}}=f(z), z=q u
$$

We find for $m=1,(n=3)$

$$
F(1,4,5 / 2, z)=(1-z)^{-2}\left[\frac{3}{16} f(z) z^{-1}+\frac{7}{8}-\frac{3}{16} z\right]
$$

and somewhat more complicated expressions for $m=2$ and $m=3$. The expressions for the full $\psi(u)$ has a similar structure. For the isochrone model, see KK68a. The complications for arbitrary $q$ are not very essential.

The parameter $q$, which characterizes central concentration, also affects the velocity distribution of the models. For moderate values of $q$, the velocity distribution function in the central part of the model is close to Maxwellian. For larger values of $q$ it becomes steeper. The parameter $p$, on the other hand, is a purely kinematic one. The radial elongation of the velocity distribution becomes larger when $p$ increases and its central concentration becomes smaller.

The case $\mu=\frac{7}{2}$ is of some interest too. The integral equation for the function $\psi(u)$ is more complicated but the expression for the relation of the radial to transversal velocity dispersions is found to be very simple:

$$
\frac{\sigma_{r}^{2}}{\sigma_{t}^{2}}=1+(\lambda+\rho \Phi) r^{2} .
$$

\section{Translator notes.}

The above study was further developed in a series of papers by Veltmann (1979b, $1981,1983)$. The distribution functions for generalized isochronous models (9), the two-parameter generalized isochronous models (see below) and the spherical models with the density law $\rho(r)=\rho_{0}\left[1+\left(r / r_{0}\right)^{2}\right]^{-\beta}$ were studied there. In particular the limit values for $\xi=0$ and asymptotics for $\xi \rightarrow \infty$ were found for the distribution function of the form $\Psi(x, \xi)=(x-\lambda \xi)^{\alpha} \psi(\xi)$ (suggested earlier by Veltmann) and the explicit expressions for the coefficients $\psi_{m, n}, g_{m, n}$ in (6), (7) were given there (if $n=3, m=0.5$ ) (Veltmann 1979b, 1981). Also the distribution function in the form

$$
\Psi(\kappa, \xi)=\sum_{m, n} a_{m, n} \kappa^{n-\frac{3}{2}}(1+2 \xi)^{-1-m-n}, \quad n \leq 4
$$

was considered by Veltmann (1981). If $\lambda=0$ then the distribution function of the generalized isochronous models is expressed in elementary functions (Veltmann, 1983).

The case $p=0$ corresponds to systems with an ellipsoidal velocity distribution (Merrit, 1985; Ossipkov, 1979; Turakulov, 1983). The explicit expression of $\psi(u)$ in 
this case was given by Malasidze (1987) for generalized isochronous models. The radial velocity dispersion was also found by him for $a \rightarrow 0$ (Parenago's potential), $a=1$ (Schuster-Plummer's sphere), and $a \rightarrow \infty$.

Generalized isochronous models with the potential (9) were described by Kuzmin \& Veltmann (1973) (see also Veltmann, 1979a) with ore details. Earlier the similar potential was used by Kuzmin \& Malasidze (1969) for the study of plane galactic orbits. Some properties of these spherical models were also described by Ossipkov (1978), who has shown the stability of such models with the spherical velocity distribution (basing on Antonov's condition $d^{3} \rho / d \Phi^{3}>0$. The model $a \rightarrow \infty$ was also discovered by Hernquist (1990) who showed that it can approximate de Vaucouleurs' density profile. Again based on the potential (9), Kuzmin \& Malasidze (1987) have constructed non-spherical models admitting a third and quadratic integral.

Veltann $(1979 \mathrm{a}, \mathrm{b})$ has considered the two-parameter generalization of the isochronous model with the potential

$$
\Phi(r)=\Phi_{0} a\left(b^{c}+\zeta^{c}\right)^{-\frac{1}{c}}, \quad a / b<1, \quad \Phi(r)=\Phi_{0}\left[1+\left(r / r_{0}\right)^{c}\right]^{-\frac{1}{c}}, \quad a / b=1 .
$$

Malasidze (1981) has suggested another generalization. His potential consists of two terms: equation (9) and the square of (9) taken with various weights. Malasidze (1984) constructed the corresponding spherical models and showed their stability (according to Antonov's criterion).

At last we have to mention that Kuzmin et.al. (1986) studied the spherical models of mass distribution with the potential $\Phi(r)=\Phi_{0} q^{-1} \ln (1+b / \zeta)$ where $\zeta(r)$ is taken according to $(9)$. The limiting case $q=1$ coincides with Jaffe's model (1983).

\section{References}

Camm, G. L., 1952 MNRAS, 112, 155.

Eddington, A. S., 1916, $M N, \mathbf{7 6}, 572$.

Herquist, L., 1990, Ap. J., 356, 359-364.

Idlis, G.M., 1961, Structurai dinamika zwiozdnykhsistem. Izd. Akad. Nauk Kaz.SSR, Alma-Ata (Trudy Astrofyz. Inst. 1.

Jaffe, W., 1983, MN, 202, 995-999.

Kuzmin, G. G., Malasidze, G. A., 1969, Tartu Publ., 38, 181-250.

Kuzmin, G. G., Malasidze, G. A., 1987, Tartu Publ., 52, 48-63.

Kuzmin, G. G., Veltmamn, Ü.-I. K., 1968a, Tartu Publ., 36, 470.

Kuzmin, G. G., Veltmann, Ü.-I. K., 1968b, Tartu Publ., 36, 3.

Kuzmin, G. G., Veltmamn, Ü.-I. K., 1973, Tartu Publ., 40, 281-323.

Kuzmin, G. G., Veltmann, Ü.-I. K., Tenjes, P. L., 1986, Tartu Publ., 51, 232-242.

Malasidze, G. A., 1981 Bull. Acad. Sci. Georgian SSR, 102, 333-336.

Malasidze, G. A., 1984 Proc. Georgian Politech. inst., 9, no 279, 118-124.

Malasidze, G. A., 1997 Abastumani Bull., 63, 115-162.

Merrit, D., 1985, $A J$, 50, 1027-1037.

Ossipkov, L. P., 1978, Astrofiz., 14, 225-237.

Ossipkov, L. P., 1979, Pis'ma v Astron. Zh., 5, 77-80.

Turakulov, Z. Ya., 1983, Astrofiz, 19, 791-801.

Veltmann Ü.-I. K., 1979a, Astron. Zh. 56, 976-980.

Veltmann Ü.-I. K., 1979b,in Star clusters (ed. K. A. Barkhatova), Ural Univ. Press, Sverdlovsk, p. 50-71.

Veltmann Ü.-I. K., 1981, Tartu Publ. 48, 232-261 (in English!).

Veltmann Ü.-I. K., 1983, Astron. Zh. 60, 223-226. 